\title{
COVID-19 and Liver Dysfunction: Current Insights and Emergent Therapeutic Strategies
}

\author{
Gong Feng ${ }^{\# 1}$, Kenneth I. Zheng ${ }^{\# 2}$, Qin-Qin Yan ${ }^{1}$, Rafael S. Rios ${ }^{2}$, Giovanni Targher ${ }^{3}$, \\ Christopher D. Byrne ${ }^{4}$, Sven Van Poucke ${ }^{5}$, Wen-Yue Liu ${ }^{6}$ and Ming-Hua Zheng*2,7,8
}

\begin{abstract}
${ }^{1}$ Xi'an Medical University, Xi'an, China; ${ }^{2}$ NAFLD Research Center, Department of Hepatology, the First Affiliated Hospital of Wenzhou Medical University, Wenzhou, China; ${ }^{3}$ Section of Endocrinology, Diabetes and Metabolism, Department of Medicine, University and Azienda Ospedaliera Universitaria Integrata of Verona, Verona, Italy; ${ }^{4}$ Southampton National Institute for Health Research Biomedical Research Center, University Hospital Southampton, Southampton General Hospital, Southampton, UK; ${ }^{5}$ Ziekenhuis Oost-Limburg, Department of Anesthesiology, Critical Care, Emergency Medicine and Pain Therapy, Genk, Belgium;

${ }^{6}$ Department of Endocrinology, the First Affiliated Hospital of Wenzhou Medical University, Wenzhou, China; ${ }^{7}$ Institute of Hepatology, Wenzhou Medical University, Wenzhou, China; ${ }^{8}$ Key Laboratory of Diagnosis and Treatment for The Development of
\end{abstract} Chronic Liver Disease in Zhejiang Province, Wenzhou, China

\begin{abstract}
The outbreak of coronavirus disease 2019 (COVID-19), caused by the severe acute respiratory syndrome coronavirus 2 (SARS-CoV-2), has attracted increasing worldwide attention. Cases of liver damage or dysfunction (mainly characterized by moderately elevated serum aspartate aminotransferase levels) have been reported among patients with COVID- 19 . However, it is currently uncertain whether the COVID-19related liver damage/dysfunction is due mainly to the viral infection per se or other coexisting conditions, such as the use of potentially hepatotoxic drugs and the coexistence of systemic inflammatory response, respiratory distress syndromeinduced hypoxia, and multiple organ dysfunction. Based on the current evidence from case reports and case series, this review article focuses on the demographic and clinical characteristics, potential mechanisms, and treatment options for COVID-19related liver dysfunction. This review also describes the geographical and demographic distribution of COVID-19-related liver dysfunction, as well as possible underlying mechanisms linking COVID-19 to liver dysfunction, in order to facilitate future drug development, prevention, and control measures for COVID-19.
\end{abstract}

Citation of this article: Feng G, Zheng KI, Yan QQ, Rios RS, Targher G, Byrne CD, et al. COVID-19 and liver dysfunction: Current insights and emergent therapeutic strategies. J Clin Transl Hepatol 2020;8(1):18-24. doi: 10.14218/ JCTH. 2020.00018.

\section{Introduction}

Severe acute respiratory syndrome coronavirus 2 (SARSCoV-2) has become a serious threat to global public health. ${ }^{1,2}$ Although the virus appears to be only partially similar to severe acute respiratory syndrome coronavirus and Middle East respiratory syndrome coronavirus, all of these viral infections are responsible for severe and potentially lethal acute respiratory syndromes in humans. ${ }^{3}$ Unfortunately, to date, there are no specific/targeted drugs, or vaccines, and the number of SARS-CoV-2-positive patients is growing in many parts of the world. The world and China map showing the geographical distribution of coronavirus disease 2019 (COVID-19) is shown in Figure 1 ; the cut-off date for this data extraction was March 5, 2020. ${ }^{4}$

Surprisingly, in addition to the acute respiratory symptoms, patients with COVID-19 also have varying degrees of liver damage/dysfunction. For example, Chen et al. ${ }^{5}$ showed that more than a third of COVID-19 patients have some liver function test abnormalities. Most of these infected patients had mild-to-moderate elevations of serum alanine aminotransferase (ALT) or aspartate aminotransferase (AST) levels; one patient had severely elevated serum aminotransferases (ALT of $7590 \mathrm{U} / \mathrm{L}$, AST of $1445 \mathrm{U} / \mathrm{L}$ ). ${ }^{5}$ Since COVID-19related liver dysfunction is now attracting widespread attention, this review discusses the epidemiological characteristics, the potential mechanisms, and the treatment options for liver dysfunction induced by COVID-19.

Epidemiological characteristics of COVID-19-related liver dysfunction

To better understand the distribution of COVID-19-related liver dysfunction in different regions of the world, we searched the available literature between December 11, 2019 and February 20, 2020. The literature search focused on initial case reports and case series covering COVID-19 patients, with a clear description of liver function tests and results. All case reports without any data on patients' liver function tests were excluded. Finally, we included 14 eligible studies, including 5 case reports (Table 1 ) and 9 case series (Table 2)..$^{5-18}$ By reviewing these 14 studies, we have 
Feng G. et al: COVID-19 and liver dysfunction
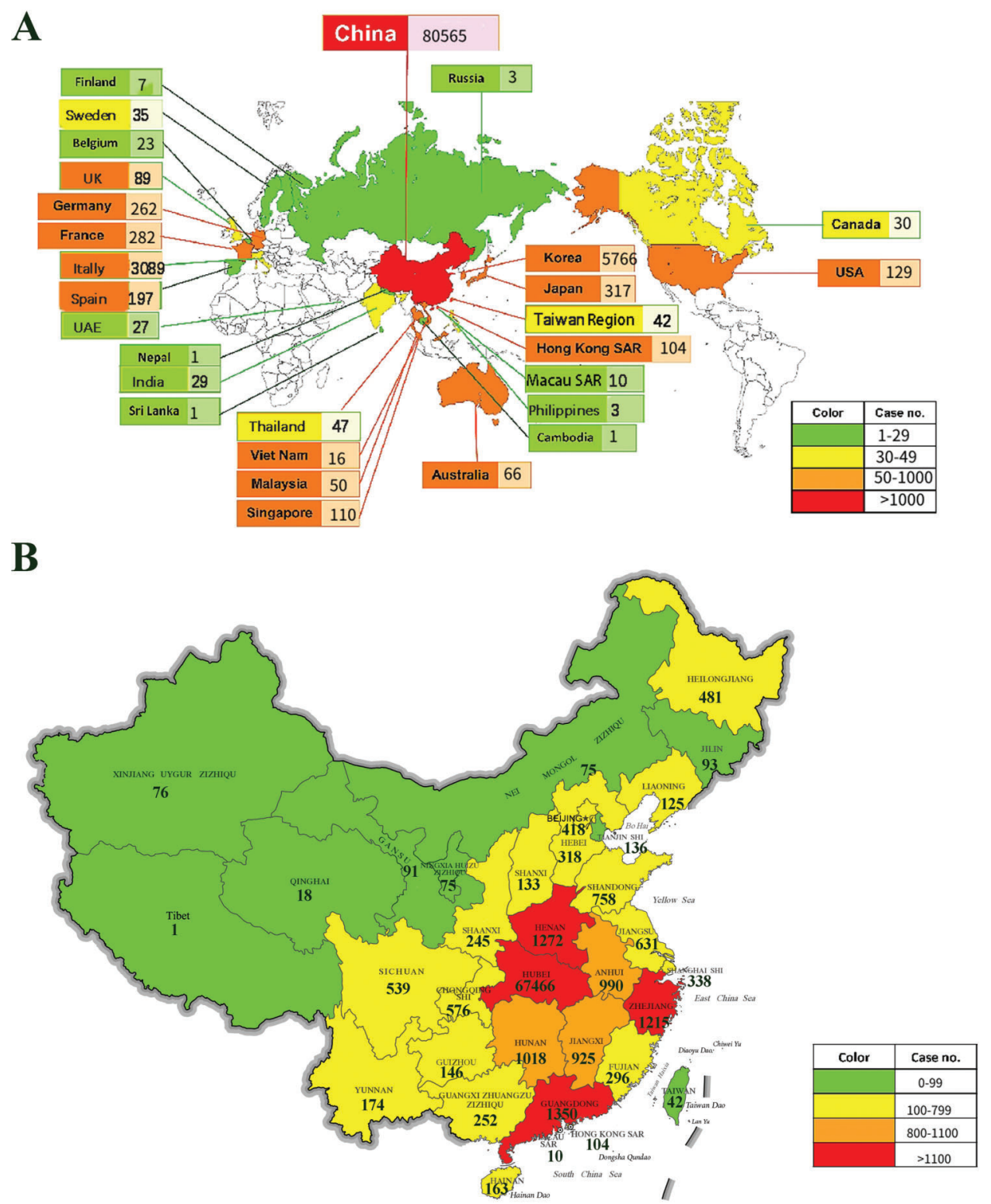

Fig. 1. Geographical distribution of COVID-19, using the cut-off date for data extraction of March 5, 2020.

described geographical and demographic characteristics of COVID-19-related liver dysfunction (as summarized below).

\section{Geographical distribution of COVID-19-related liver dysfunction}

In two Chinese multicenter surveys, one of which was led by the First Affiliated Hospital of Guangzhou Medical University, including 552 hospitals in 31 provinces/provincial municipalities through January 29th, 2020 and the other was led by the First Hospital of Lanzhou University including 7 hospitals, a considerable number of patients with COVID-19 had some abnormalities in liver function tests. ${ }^{11,16}$ In particular, $6.2 \%$ to $22.2 \%$ of patients had increased serum AST levels (Fig. 2) and $21.3 \%$ to $28.1 \%$ of patients had increased serum ALT levels, respectively. ${ }^{11,16}$ of the six studies performed in Wuhan, China, $5,13-15,17,18$ only four included data on the proportion of patients with abnormal liver function test results. Specifically, in these four studies, the proportion of infected patients with increased serum AST levels ranged from $24.1 \%$ to $36.6 \%$ (Fig. 2)..$^{5,13,17,18}$ In a survey from Zhejiang Province, China, the proportion of patients with increased serum AST levels was only $16.1 \%$, whereas the proportion of those with increased serum ALT levels was not specified. ${ }^{12}$ It seems likely that the proportion of infected patients with increased serum AST levels in Wuhan (i.e. the area in which the epidemic of COVID-19 started) is much greater than cases reported outside Wuhan. It is plausible to speculate that there may have been a higher viral load of COVID-19 in 
Feng G. et al: COVID-19 and liver dysfunction

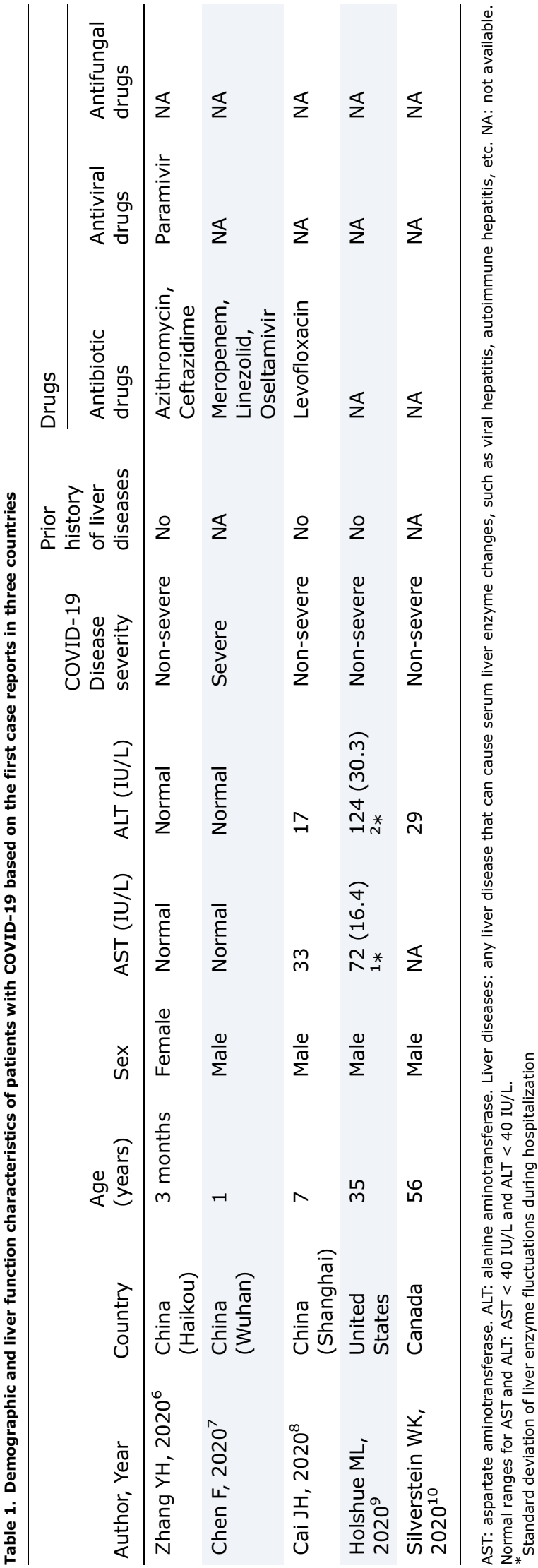

exposed patients in Wuhan, where the infection began and was concentrated in a greater proportion of the population.

\section{Sex distribution of COVID-19-related liver dysfunction}

A total of six case series reporting the percentage of abnormal liver function test results amongst COVID-19 patients suggested that the proportion of infected men with increased serum AST levels was higher than that observed in infected women. ${ }^{5,11-13,16,17}$ In fact, in these case series, the proportions of infected men with increased serum AST levels were, respectively, $68.7 \%, 58.2 \%, 58.1 \%, 72.4 \%, 62.8 \%$ and $73.2 \%$, whereas the proportions of infected women were, respectively, $31.3 \%, 41.8 \%, 41.9 \%, 27.6 \%, 37.2 \%$ and $26.8 \%$. It is possible to hypothesize that infected men are more predisposed to develop COVID-19-associated liver dysfunction than infected women, and we suggest that further research is required to better understand this sex-related difference.

\section{Age distribution of COVID-19-related liver dysfunction}

Of the five case reports, three were in children and two were in adults. The age of children ranged from 3 months to 7 years, whereas the age of adult patients ranged from 35 to 56 years. None of these children had abnormal serum liver enzymes and, therefore, it is possible to hypothesize that older age is associated with a higher likelihood of liver damage/dysfunction. However, further studies are needed to confirm this finding. We look forward to more case reports/ case series on COVID-19-related liver damage/dysfunction in different age groups in the future.

Putative mechanisms of COVID-19-related liver dysfunction

\section{Angiotensin-converting enzyme (ACE)2-mediated liver dysfunction}

Whether SARS-CoV-2 has direct adverse effects on liver function is currently not known. Some studies have suggested that SARS-CoV-2 predominantly enters alveolar epithelial cells through the human ACE2 receptor. ${ }^{19,20}$ Therefore, the lung is considered the main target organ of SARS-CoV-2 infection. However, previous studies have found that bile duct epithelial cells may also express ACE2 receptor at a concentration 20 times higher than in hepatocytes and these findings suggest that SARS-CoV-2 infection might also cause bile duct epithelial cell damage. ${ }^{21,22}$ However, significant increases in circulating levels of serum alkaline phosphatase, bilirubin or gamma-glutamyltransferase (that may reflect bile duct injury) have been rarely reported in COVID-19 patients. ${ }^{9}$ Liver histopathologic features from COVID-19 patients also did not show any significant damage in hepatocytes or bile duct cells. ${ }^{23}$ For this reason, it is reasonable to assume that COVID-19-related liver dysfunction is more likely due to secondary liver damage than the use of hepatotoxic therapies or the coexistence of systemic inflammatory response, respiratory distress syndrome-induced hypoxia, or multiple organ dysfunction. 


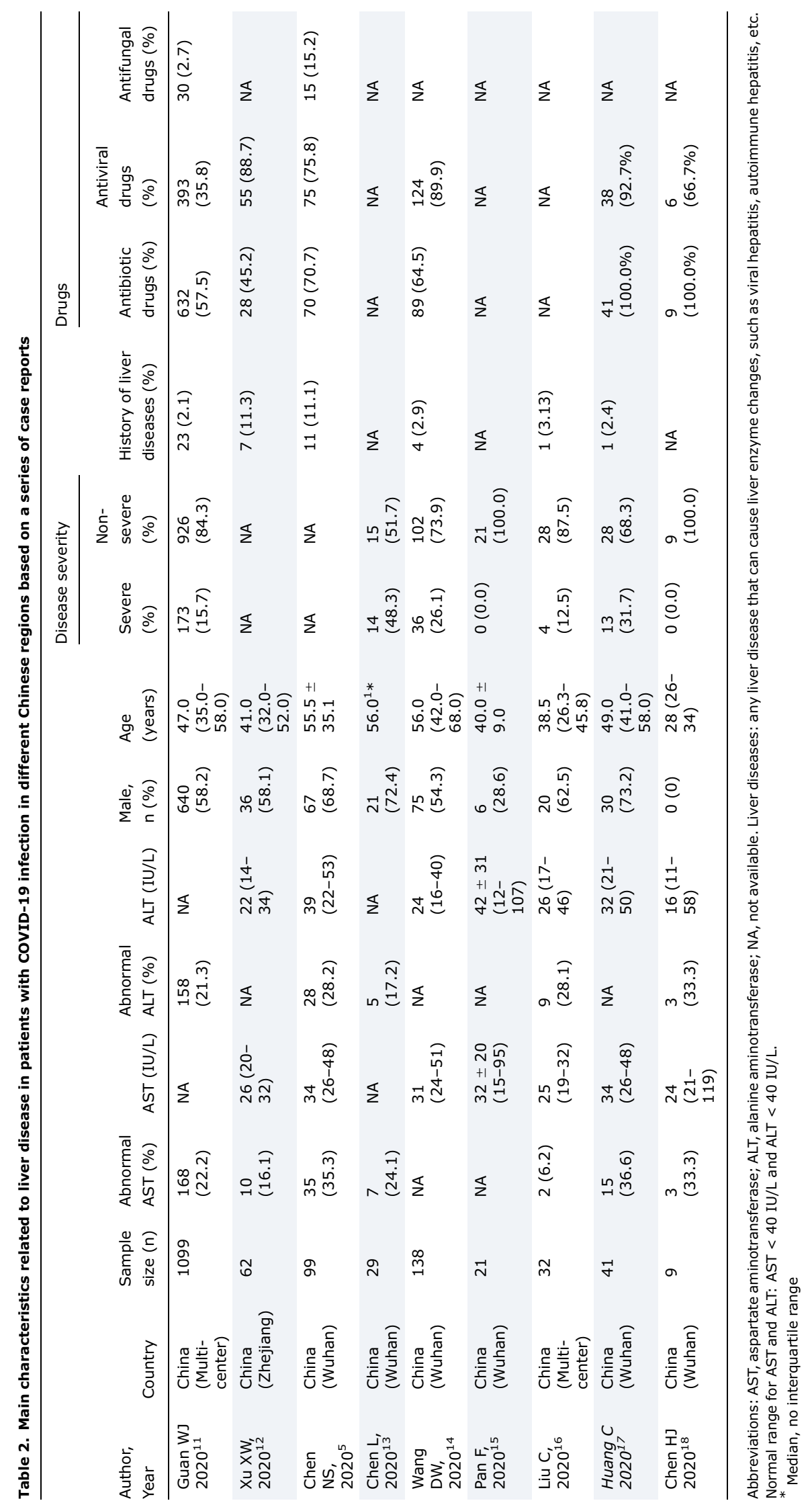


Feng G. et al: COVID-19 and liver dysfunction

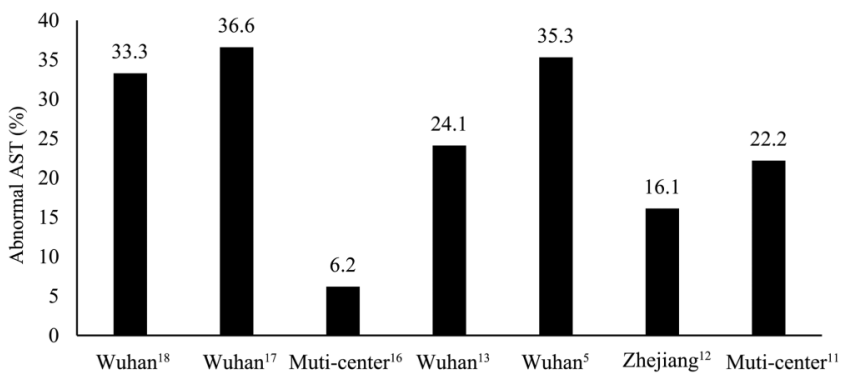

Fig. 2. Proportion of patients with liver dysfunction in Chinese regions: Wuhan and outside Wuhan.

\section{Drugs}

During the course of the COVID-19 epidemic, many infected patients have been treated with antipyretic agents. Most of these medications contain acetaminophen, which is a drug recognized as being able to cause significant liver damage or induce liver failure. ${ }^{23}$ It is known that an acute ingestion of $>7.5$ to $10 \mathrm{~g}$ of acetaminophen in adults or 150 to $200 \mathrm{mg} / \mathrm{kg}$ in children is likely to cause hepatotoxicity. ${ }^{24}$ Although the US Food and Drug Administration Advisory Committee has proposed a decrease in the maximum daily dosage of acetaminophen from 4 to $3 \mathrm{~g}$, and the maximum individual dosage from 1 to $0.65 \mathrm{~g}$, (relegating $500-\mathrm{mg}$ tablets to prescription status), these recommendations have not been implemented worldwide. ${ }^{25}$ In addition, although there is currently no targeted antiviral treatment for COVID-19, many infected patients have also been treated with some antiviral drugs, such as oseltamivir, abidol or lopinavir, which may have some hepatotoxic effects.

\section{Systemic inflammatory response syndrome}

Although many COVID-19 patients were not too unwell, this infection in some patients has resulted in sudden deterioration, ending in multiple organ failure. Most experts believe that the occurrence of multiple organ failure is mainly related to the sudden initiation of an inflammatory "storm" in the critically ill COVID-19 patients. ${ }^{26}$ The so-called inflammatory "storm", or systemic inflammatory response syndrome, is strongly related to activation of both natural and cellular immunity that is triggered by COVID-19 infection. ${ }^{27}$ In fact, the virus is able to directly induce multiple proinflammatory signals via toll-like receptors (TLRs) and activation of killer T lymphocytes. ${ }^{28}$ The activated $T$ lymphocytes then attack the infected body cells, leading to their apoptosis and necrosis, until $\mathrm{T}$ lymphocytes are depleted. Damage-related pattern molecules released by dead infected cells can further amplify some inflammatory signals, such as TLRs. At the same time, T-lymphocyte depletion cannot control viral and bacterial infections, thereby activating multiple inflammatory signaling pathways, which lead to macrophage activation and secondary inflammatory reactions. Subsequently, when more inflammatory cytokines are released, more cell damage and necrosis are observed (Fig. 3). Such a vicious cycle is capable of causing multiple injuries, not only to the lungs but also to the liver, heart, and kidneys.

\section{Hypoxia-reperfusion dysfunction}

Hypoxia and shock induced by COVID-19-related complications (such as respiratory distress syndrome, systemic inflammatory response syndrome, and multiple organ failure) may also cause hepatic ischemia and hypoxia-reperfusion dysfunction. Experimental data showed that hepatic cell death and inflammatory cell infiltration caused by hypoxia can be seen both in vivo and in vitro models of hepatic ischemia and hypoxia. ${ }^{29}$ This suggests that oxygen reduction and lipid accumulation in hepatocytes during shock and hypoxic conditions may lead to cell death. The subsequent marked increase in reactive oxygen species and their peroxidation products can act as a second messenger, activating redoxsensitive transcription factors, and further amplifying the release of multiple proinflammatory factors, causing liver damage. ${ }^{30}$ All the aforementioned findings suggest that pneumonia-associated hypoxia is one of the most important factors causing secondary liver injury in COVID-19 patients.

In summary, the COVID-19-related liver dysfunction may be considered as the result of secondary liver damage caused mainly by several factors, such as the use of potentially hepatotoxic drugs, systemic inflammatory response, respiratory distress syndrome-induced hypoxia, and multiple organ failure. In addition, critically ill COVID-19 patients with severe liver dysfunction are also more likely to have a poorer prognosis.

\section{Treatment options for COVID-19-related liver dysfunction}

Presently, there is no specific treatment for COVID-19 infection. ${ }^{31}$ Therefore, the cornerstone of COVID-19 management is patient isolation and supportive medical care where necessary, including pulmonary ventilation and prevention of the underlying inflammatory "storm" as well. ${ }^{32}$

From the findings discussed above, however, we believe that it is also reasonable to explore novel treatments for COVID-19 targeting of the ACE2 receptor. The ACE2 cellular receptor is highly expressed in human lung tissues, gastrointestinal tract, liver, vascular endothelial cells, and arterial smooth muscle cells. ${ }^{33}$ In addition, skin, nasal cavity, and oral mucosa basal cells also express the ACE2 receptor. ${ }^{27}$ All organs with high expression of the ACE2 receptor may be targeted by SARS-CoV-2 infection. ${ }^{34}$ Activation of the ACE2/Ang

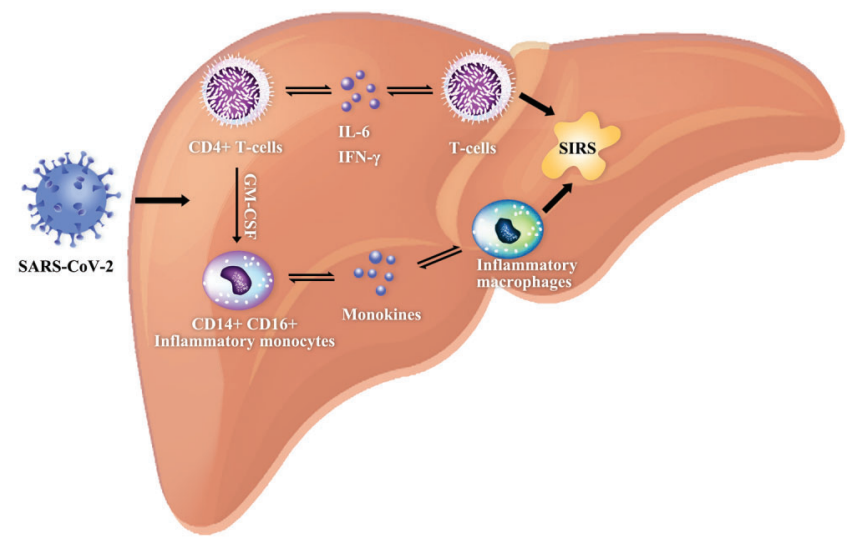

Fig. 3. Schematic diagram showing the systemic inflammatory response syndrome induced by SARS-CoV2. After the SARS-CoV- 2 infection, pathogenic $T$ cells are rapidly activated, producing granulocyte-macrophage colony-stimulating factor (GM-CSF), interleukin (IL)- 6 and other proinflammatory factors. GM-CSF will further activate $\mathrm{CD} 14^{+} \mathrm{CD} 16^{+}$inflammatory monocytes, producing a larger amount of IL- 6 and other proinflammatory factors, and thereby inducing an inflammatory "storm" that leads to immune damage to other organs, such as the lungs and the liver. Both IL-6 and GM-CSF are two key proinflammatory factors that trigger the inflammatory "storm" in patients with COVID-19. 
(1-7)/Mas signaling pathway or inhibition of the ACE/Ang II/ AT1R pathway could be potential pathways for the treatment of COVID-19. For SARS-CoV-2-infected patients, both ACE-inhibitors and angiotensin-II-receptor antagonists might be used not only for treating high blood pressure but also for reducing systemic inflammatory response and improving patient mortality. ${ }^{35}$

Recently, Chen et al. ${ }^{36}$ reported that glycyrrhizic acid derivatives might also have antiviral activity against SARS-CoV-2. Glycyrrhizic acid is one of the first-line drugs for anti-inflammatory protection in liver disease, and it has been used in clinical practice for many years. ${ }^{37}$ In particular, glycyrrhizic acid is a triterpene glycoside isolated from the root of the licorice plant. ACE2 is a cellular type I membrane protein that is mostly expressed in the lungs, heart, kidneys, and intestine. Full-length ACE2 consists of an N-terminal peptidase domain and a C-terminal collectrin-like domain that ends with a single trans-membrane helix and a $\sim 40$-residue intracellular segment. ${ }^{38}$ Glycyrrhizin has the potential to bind to ACE2 receptor with an estimated $\Delta G(\mathrm{kcal} / \mathrm{mol})$ of -9 , with the binding sites of ARG-559, GLN-388, ARG-393, and ASP-30. ${ }^{36}$

\section{Conclusions}

Our review shows the following: (1) In highly epidemic areas of COVID-19 infection, such as Wuhan, China, the proportion of infected patients with abnormal liver function test results (mainly elevated serum AST levels) is greater than that observed in regions where a smaller proportion of cases of COVID-19 infection in the population have occurred. (2) The proportion of infected patients with elevated serum transaminase levels is higher in adults than in children and in men than in women, respectively. However, we suggest that further studies are needed to confirm these preliminary observations. In the meantime, we believe that the front-line medical staff should pay attention to liver function tests in patients infected with COVID-19. For those patients with a pre-existing history of liver diseases (especially older patients), special attention should be paid to monitoring hepatic changes caused by COVID-19, whilst carefully identifying the cause of the liver dysfunction. ${ }^{39}$ We also recommend that front-line medical staff should assess the use of appropriate hepatoprotective therapies, especially in patients with pre-existing liver disease, in order to attenuate the potentially deleterious impact of COVID-19-related liver damage/dysfunction.

\section{Funding}

This work was supported by grants from the National Natural Science Foundation of China (81500665), High Level Creative Talents from Department of Public Health in Zhejiang Province and Project of New Century 551 Talent Nurturing in Wenzhou. GT is supported in part by grants from the University School of Medicine of Verona, Verona, Italy. CDB is supported in part by grants from the Southampton National Institute for Health Research Biomedical Research Centre (IS-BRC-20004), UK.

\section{Conflict of interest}

The authors have no conflict of interests related to this article.

\section{Author contributions}

Design and data interpretation (MHZ, GF, KIZ, QQY, WYL), manuscript writing (GF, KIZ, RSR), critical revision of the manuscript (MHZ, GT, CDB, SVP). All authors reviewed and commented on the manuscript and approved the final version.

\section{References}

[1] Zhu N, Zhang D, Wang W, Li X, Yang B, Song J, et al. A novel coronavirus from patients with pneumonia in China, 2019. N Engl J Med 2020;382:727-733. doi: 10.1056/NEJMoa2001017.

[2] Li Q, Guan X, Wu P, Wang X, Zhou L, Tong Y, et al. Early transmission dynamics in Wuhan, China, of novel coronavirus-infected pneumonia. N Engl J Med 2020. doi: 10.1056/NEJMoa2001316.

[3] Zhang C, Shi L, Wang FS. Liver injury in COVID-19: management and challenges. Lancet Gastroenterol Hepatol 2020. doi: 10.1016/S2468-1253(20) 30057-1.

[4] WHO. Coronavirus disease 2019 (COVID-19) situation report-49. 2020. Available from: https://www.who.int/docs/default-source/coronaviruse/situation-reports/20200309-sitrep-49-covid-19.pdf?sfvrsn=70dabe61_4

[5] Chen N, Zhou M, Dong X, Qu J, Gong F, Han Y, et al. Epidemiological and clinical characteristics of 99 cases of 2019 novel coronavirus pneumonia in Wuhan, China: a descriptive study. Lancet 2020;395:507-513. doi: 10. 1016/S0140-6736(20)30211-7.

[6] Zhang YH, Lin DJ, Xiao MF, Wang JC, Wei Y, Lei ZX, et al. [2019-novel coronavirus infection in a three-month-old baby]. Zhonghua Er Ke Za Zhi 2020; 58:E006. doi: 10.3760/cma.j.issn.0578-1310.2020.0006.

[7] Chen F, Liu ZS, Zhang FR, Xiong RH, Chen Y, Cheng XF, et al. [First case of severe childhood novel coronavirus pneumonia in China]. Zhonghua Er Ke Za Zhi 2020;58:E005. doi: 10.3760/cma.j.issn.0578-1310.2020.0005.

[8] Cai JH, Wang XS, Ge YL, Xia AM, Chang HL, Tian H, et al. [First case of 2019 novel coronavirus infection in children in Shanghai]. Zhonghua $\mathrm{Er} \mathrm{Ke} \mathrm{Za} \mathrm{Zhi}$ 2020;58:86-87. doi: 10.3760/cma.j.issn.0578-1310.2020.02.002.

[9] Holshue ML, DeBolt C, Lindquist S, Lofy $\mathrm{KH}$, Wiesman J, Bruce $\mathrm{H}$, et al. First case of 2019 novel coronavirus in the United States. N Engl J Med 2020;382: 929-936. doi: 10.1056/NEJMoa2001191.

[10] Silverstein WK, Stroud L, Cleghorn GE, Leis JA. First imported case of 2019 novel coronavirus in Canada, presenting as mild pneumonia. Lancet 2020; 395:734. doi: 10.1016/S0140-6736(20)30370-6.

[11] Guan WJ, Ni ZY, Hu Y, Liang WH, Ou CQ, He JX, et al. Clinical characteristics of coronavirus disease 2019 in China. N Engl J Med 2020. doi: 10. 1056/NEJMoa2002032.

[12] $X u X W, W u X X$, Jiang XG, $X u$ KJ, Ying $L J, M a C L$, et al. Clinical findings in a group of patients infected with the 2019 novel coronavirus (SARS-Cov-2) outside of Wuhan, China: retrospective case series. BMJ 2020;368:m606. doi: $10.1136 / \mathrm{bmj} . \mathrm{m} 606$

[13] Chen L, Liu HG, Liu W, Liu ], Liu K, Shang J, et al. [Analysis of clinical features of 29 patients with 2019 novel coronavirus pneumonia]. Zhonghua Jie $\mathrm{He} \mathrm{He}$ Hu Xi Za Zhi 2020;43:E005. doi: 10.3760/cma.j.issn.1001-0939.2020.0005.

[14] Wang D, Hu B, Hu C, Zhu F, Liu X, Zhang J, et al. Clinical characteristics of 138 hospitalized patients with 2019 novel coronavirus-infected pneumonia in Wuhan, China. JAMA 2020. doi: 10.1001/jama.2020.1585.

[15] Pan $F$, Ye T, Sun $P$, Gui $S$, Liang $B$, Li L, et al. Time course of lung changes on chest CT during recovery from 2019 novel coronavirus (COVID-19) pneumonia. Radiology 2020;200370. doi: 10.1148/radiol.2020200370.

[16] Liu C, Jiang ZC, Shao CX, Zhang HG, Yue HM, Chen ZH, et al. [Preliminary study of the relationship between novel coronavirus pneumonia and liver function damage: a multicenter study]. Zhonghua Gan Zang Bing Za Zhi 2020;28:148-152. doi: 10.3760/cma.j.issn.1007-3418.2020.02.003.

[17] Huang C, Wang Y, Li X, Ren L, Zhao J, Hu Y, et al. Clinical features of patients infected with 2019 novel coronavirus in Wuhan, China. Lancet 2020;395: 497-506. doi: 10.1016/S0140-6736(20)30183-5.

[18] Chen H, Guo J, Wang C, Luo F, Yu X, Zhang W, et al. Clinical characteristics and intrauterine vertical transmission potential of COVID-19 infection in nine pregnant women: a retrospective review of medical records. Lancet 2020; 395:809-815. doi: 10.1016/S0140-6736(20)30360-3.

[19] Li R, Qiao S, Zhang G. Analysis of angiotensin-converting enzyme 2 (ACE2) from different species sheds some light on cross-species receptor usage of a novel coronavirus 2019-nCoV. J Infect 2020. doi: 10.1016/j.jinf.2020.02. 013

[20] Xu H, Zhong L, Deng J, Peng J, Dan H, Zeng X, et al. High expression of ACE2 receptor of 2019-nCoV on the epithelial cells of oral mucosa. Int J Oral Sci 2020;12:8. doi: 10.1038/s41368-020-0074-x

[21] Chai X, Hu L, Zhang Y, Han W, Lu Z, Ke A, et al. Specific ACE2 expression in cholangiocytes may cause liver damage after 2019-nCoV infection. bioRxiv 2020(v1). doi: 10.1101/2020.02.03.931766.

[22] Guan GW, Gao L, Wang JW, Wen X], Mao TH, Peng SW, et al. [Exploring the mechanism of liver enzyme abnormalities in patients with novel coronavirusinfected pneumonia]. Zhonghua Gan Zang Bing Za Zhi 2020;28:E002. doi: 10.3760/cma.j.issn.1007-3418.2020.02.002. 
[23] Xu Z, Shi L, Wang Y, Zhang J, Huang L, Zhang C, et al. Pathological findings of COVID-19 associated with acute respiratory distress syndrome. Lancet Respir Med 2020. doi: 10.1016/s2213-2600(20)30076-x.

[24] Hodgman MJ, Garrard AR. A review of acetaminophen poisoning. Crit Care Clin 2012;28:499-516. doi: 10.1016/j.ccc.2012.07.006.

[25] Krenzelok EP. The FDA Acetaminophen Advisory Committee Meeting - what is the future of acetaminophen in the United States? The perspective of a committee member. Clin Toxicol (Phila) 2009;47:784-789. doi: 10. 1080/15563650903232345.

[26] Prompetchara E, Ketloy C, Palaga T. Immune responses in COVID-19 and potential vaccines: Lessons learned from SARS and MERS epidemic. Asian Pac J Allergy Immunol 2020;38:1-9. doi: 10.12932/ap-200220-0772.

[27] Klimstra WB, Ryman KD, Bernard KA, Nguyen KB, Biron CA, Johnston RE. Infection of neonatal mice with sindbis virus results in a systemic inflammatory response syndrome. J Virol 1999;73:10387-10398.

[28] Tartey S, Takeuchi O. Pathogen recognition and Toll-like receptor targeted therapeutics in innate immune cells. Int Rev Immunol 2017;36:57-73. doi: 10.1080/08830185.2016.1261318.

[29] Yang L, Wang W, Wang X, Zhao J, Xiao L, Gui W, et al. Creg in hepatocytes ameliorates liver ischemia/reperfusion injury in a TAK1-dependent manner in mice. Hepatology 2019;69:294-313. doi: 10.1002/hep.30203.

[30] Zhang XJ, Cheng X, Yan ZZ, Fang J, Wang X, Wang W, et al. An ALOX12-12HETE-GPR31 signaling axis is a key mediator of hepatic ischemia-reperfusion injury. Nat Med 2018;24:73-83. doi: 10.1038/nm.4451.
[31] Zhang Z, Li X, Zhang W, Shi ZL, Zheng Z, Wang T. Clinical features and treatment of 2019-nCov pneumonia patients in Wuhan: report of a couple cases. Virol Sin 2020. doi: 10.1007/s12250-020-00203-8.

[32] Zhang L, Liu Y. Potential interventions for novel coronavirus in China: A systematic review. J Med Virol 2020;92:479-490. doi: 10.1002/jmv.25707.

[33] Santos RAS, Sampaio WO, Alzamora AC, Motta-Santos D, Alenina N, Bader $M$, et al. The ACE2/Angiotensin-(1-7)/MAS axis of the renin-angiotensin system: focus on angiotensin-(1-7). Physiol Rev 2018;98:505-553. doi: 10.1152/physrev.00023.2016.

[34] Ding Y, Wang H, Shen H, Li Z, Geng J, Han H, et al. The clinical pathology of severe acute respiratory syndrome (SARS): a report from China. J Pathol 2003;200:282-289. doi: 10.1002/path.1440.

[35] Sun ML, Yang JM, Sun YP, Su GH. [Inhibitors of RAS Might Be a Good Choice for the Therapy of COVID-19 Pneumonia]. Zhonghua Jie He He Hu Xi Za Zhi 2020;43:E014. doi: 10.3760/cma.j.issn.1001-0939.2020.0014.

[36] Chen H, Du Q. Potential natural compounds for preventing 2019-nCoV infection. Preprints 2020;2020010358(v3). doi: 10.20944/preprints202001.0358.v3.

[37] Li JY, Cao HY, Liu P, Cheng GH, Sun MY. Glycyrrhizic acid in the treatment of liver diseases: literature review. Biomed Res Int 2014;2014:872139. doi: $10.1155 / 2014 / 872139$.

[38] Yan R, Zhang Y, Li Y, Xia L, Zhou Q. Structure of dimeric full-length human ACE2 in complex with B0ATI. bioRxiv 2020(v1). doi: 10.1101/2020.02.17. 951848.

[39] Cheng Z], Shan J. 2019 novel coronavirus: where we are and what we know. Infection 2020. doi: 10.1007/s15010-020-01401-y. 